\title{
CMA opposes gender discrimination against doctors
}

\author{
Early released on Aug. 26, 2008
}

$\mathrm{T}$ he Canadian Medical Association (CMA) has voted to oppose gender discrimination against Canadian doctors and trainees stemming from patient pressure to apply practices "dictated by their religion on culture."

"A worrisome phenomenon seems to be emerging in several health care settings" which affects several medical specialties and is "due to explicitly expressed values or beliefs that are solidly entrenched in the cultures and religions of certain communities," said Quebec Medical Association President Dr. Jean-Bernard Trudeau, who introduced the controversial motion.

In Canada and in Quebec "men with deep religious commitments demand that their wives be examined by a female obstetrician-gynecologist and refuse any professional intervention by a male physician, even when safe childbirth is compromised," Trudeau said at the CMA's 141st annual general council.

"These demands go against our society's fundamental values and should not be accepted. ...All physicians are recognized as having the necessary skills to practice their profession, all share the same duty to respect their professional oath, Hippocratic Oath and code of conduct."

The issue is of particular concern to medical residents who may not get the clinical experience they need because of patient demands that exclude them from providing care, said Dr. Jimmy Bejjani, who seconded the motion.

A motion that was introduced Aug. 18, 2008, to refer the issue to the CMA board of directors was rejected by a slim margin - a 2/3 vote was required, but only $65 \%$ of delegates voted in favour. Delegates asked that it be reworded and reintroduced. It resurfaced on Aug. 20, with the word "gender" added. It passed with the approval of $80 \%$ of delegates.

Some, though, expressed skepticism about the motion's merits.

"Sometimes people put demands on us that may not be reasonable or appropriate," said Dr. Carolyn Lane of Calgary, who had opposed the motion, arguing that this issue is thorny and complex and requires careful consideration.

"I don't see this as an issue of discrimination. I would prefer a motion that supports doctors in ensuring access to care regardless of cultural or religious-based demands - a positive statement," she said in an interview.

Bejjani said that the impact of the discrimination was worsened by the doctor shortage in Quebec - especially when patients come to the emergency ward and insist they be treated by a particular gender of doctor.

Dr. Bonnie Cham, chair of the CMA Committee on Ethics, said in an interview that the motion, which she understood from conversations was meant to apply mainly to emergency care, is "open to misinterpretation." She wanted the motion referred to the board of directors.

Among the other approved delegate motions:

- "The CMA opposes adoption of Bill C-484 and of any legislation that would result in compromising access for women the medical services required to terminate a preg- nancy." The rationale is that Bill C-484 would "implicitly confer legal status on the fetus, which is not the case in current law. Adoption of the Bill could open the door to all kinds of claims or court actions calling for the recriminalization of abortion, said Dr. Paul Robinson, who put forward the motion.

- "The CMA and provincial/territorial medical associations will lobby for appropriate 'apology' legislation in all Canadian jurisdictions." The rationale behind such legislation is to allow doctors to express regret or apologize to a patient, about an adverse event, without incurring any potential medico-legal risk.

- "The CMA objects to the current practice of insurers, employers and other third parties requesting and gaining access to unlimited medical information obtained as a result of patients signing forms that grant unrestricted 'consent for release of information,' which claiming eligibility for disability benefits." The rationale is that most patients are unaware that signing such a general consent to release medical information may allow their employer or insurers to gain access to records that go back several years and may "contain very sensitive information and have questionable relevance to the issue at hand." Consent for release of information should be specific and limited to information that relates to the injury or illness at hand, explained Dr. John Tracey, who introduced the motion. — Ann Silversides, CMAJ

CMAJ 2009. DOI:10.1503/cmaj.081326 